\title{
Pediatric case of persistent hiccups associated with hypertrophic olivary degeneration
}

\author{
Pınar Arıcan $^{1 \oplus}$, Özgür Öztekin ${ }^{2 \odot}$, Dilek Çavuşoğlu ${ }^{3 \odot}$, Sema Bozkaya Yılmaz ${ }^{1 \oplus}$, \\ Atilla Ersen $^{1 \odot}$, Nihal Olgaç Dündar ${ }^{4}$, Pınar Gençpınar $^{4 \odot}$ \\ Departments of ${ }^{1}$ Pediatric Neurology and ${ }^{2}$ Radiology, Izmir Tepecik Education and Research Hospital, İzmir; ${ }^{3}$ Department of Pediatric \\ Neurology, Afyon Kocatepe University, Afyon; ${ }^{4}$ Department of Pediatric Neurology, İzmir Katip Çelebi University, İzmir, Turkey.
}

\begin{abstract}
Background. Hypertrophic olivary degeneration (HOD) is a rare degenerative disorder that is thought to occur subsequent to a disruption of the dentate-rubro-olivary pathway.

Case. We report a pediatric case of unilateral HOD presented with persistent hiccups and palatal tremor. Radiological examination of diaphragm was normal considering ultrasound and chest $\mathrm{x}$-ray. On T2WI (weighted images) and Fluid Attenuated Inversion Recovery (FLAIR) images, hyperintense enlargement of the right inferior olivary nucleus was seen. No abnormal enhancement was detected on post-contrast scans and no evidence of restricted diffusion was seen. Susceptibility weighted imaging (SWI) sequences revealed a chronic hemorrhage involving the medulla oblongata and cerebellum. Cranial magnetic resonance imaging (MRI) findings were consistent with unilateral HOD. Palatal tremor and dentate-rubral tremor are frequent presentation of HOD, however to our knowledge persistent hiccups had not yet been reported in children with HOD.

Conclusion. We highlight a pediatric case of unilateral HOD, which presented with persistent hiccups. Awareness of clinical and radiological findings of HOD is important to avoid misinterpretation as a mass lesion, an ischemic event, or a demyelinating disease and provide adequate management.
\end{abstract}

Key words: children, hiccups, hypertrophic olivary degeneration.

Hypertrophic olivary degeneration (HOD) is a transsynaptic degeneration, occurring subsequent to a lesion involving dentato-rubro-olivary pathway, also called the Guillain-Mollaret triangle. ${ }^{1}$ Any lesions involved in any components of this pathway from dentate nucleus to inferior olivary nucleus can lead to the loss of afferent signals into inferior olivary nucleus and cause HOD. ${ }^{2}$

The clinical manifestations of HOD are variable. Palatal tremor, dentate-rubral tremor, ocular myoclonus and cerebellar symptoms including ataxia are classically associated clinical findings

$\triangle$ Pınar Gençpınar

pinargencpinar@yahoo.com.tr

Received 19th November 2018, revised 28th December 2018, accepted 31st January 2019. of HOD. ${ }^{3}$ Patients also may represent no clinical manifestations associated with HOD and diagnosis of HOD can be made with routine surveillance magnetic resonance imaging (MRI). ${ }^{4}$

Rhythmic involuntary jerking movements are frequent presentation of HOD, however persistent hiccups had not yet been reported in children with HOD. We report a pediatric case of persistent hiccups associated with unilateral HOD.

\section{Case Report}

A 17-year-old male patient admitted with complaints of persistent hiccups over a period of 5 days. Two years ago, he presented with postural tremor and was diagnosed with 
unilateral HOD. He did not develop any additional clinical features of HOD such as ocular myoclonus or ataxia within two years. $\mathrm{He}$ was the fourth child of consanguineous parents with no relevant medical familial history. He was born full term after a normal pregnancy. His history revealed that he had another episode of persistent hiccups for 3 days two months ago. On physical examination, his weight, height, and head circumference were normal for his age. There were no organomegaly or dysmorphic features. Neurologic examination was normal, except for postural tremor and palatal tremor.

Hemoglobin level, serum electrolytes, renal function, liver function and thyroid function tests were normal. Radiological examination of diaphragm was normal considering ultrasound and chest $x$-ray. Right inferior olivary nucleus was enlarged and hyperintense on both T2WI (weighted images) and Fluid Attenuated Inversion Recovery (FLAIR) images, no enhancement detected after contrast administration. No evidence of restricted diffusion was seen. Susceptibility weighted imaging (SWI) sequences revealed a chronic hemorrhage involving the medulla oblongata and cerebellum. Cranial MRI findings were consistent with unilateral HOD (Fig 1). Hematologic and coagulation parameters were normal. Magnetic resonance angiography demonstrated no evidence of vascular malformation.

For further genetic investigation of possible metabolic disease, TruSight Inherited Disease Sequencing Panel (Illumina Inc.) was used. This gene panel covered 552 genes including POLG and SURF1 genes associated with HOD. Next generation sequencing analysis of TruSight Inherited Disease Sequencing Panel did not reveal a pathogenic mutation associated with the patient's phenotype. Serum ceruloplasmin level was $292 \mathrm{mg} / \mathrm{L}$ (200-600mg/L). Serum autoantibodies including antinuclear antibody, anti-double stranded DNA, HLA-B51 for systemic vasculitis were negative. A written informed consent was obtained from the parents of the patient.

\section{Discussion}

We identified a pediatric case of unilateral HOD present with persistent hiccups and palatal tremor. Hypertrophic olivary degeneration can be associated with synchronous movements of the larynx, pharynx, diaphragm, and facial muscles. ${ }^{5}$ Palatal tremor and dentatorubral tremor are the most specific rhythmic involuntary movements attributable to HOD. The exact mechanism underlying the synchronous movements remains unclear but the accepted hypothesis involves the loss of GABAergic inhibitory cerebellar afferents of the dentate-olivary tract. Olivary neurons are believed to have intrinsic oscillator property and decreased GABAergic descending inhibition leads to hypersynchronous discharge. ${ }^{6,7}$

Histologically, there is vacuolar cytoplasmic degeneration and an increase in the number of astrocytes resulting in hypertrophy of the affected inferior olivary nucleus neurons which most frequently develops weeks or months secondary to disruption of the dentate-rubroolivary tract. Which is followed by subsequent atrophy and gliosis within 3-4 years. ${ }^{3,8}$

MRI findings of HOD is classical and problem solving, in which inferior olivary nucleus has both an increased signal intensity in T2WI and FLAIR images and hypertrophic. The absence of contrast enhancement is important as it differentiates HOD from malignant tumors and inflammation and the absence of restricted diffusion differentiates from ischemic event. ${ }^{9}$

The most common causes of HOD are hemorrhage, tumor, trauma and vascular malformations. Hypertrophic olivary degeneration has been also reported in metabolic, infectious, neurodegenerative disease and metronidazole intoxication. Prognosis depends on the underlying etiology. ${ }^{10-12}$ In one in every five patients with HOD no cause can be identified. ${ }^{9}$ Resection of posterior cranial fossa tumors and cavernous angioma leading to hemorrhage which subsequently developed HOD have been reported. ${ }^{6,8}$ In our patient, 

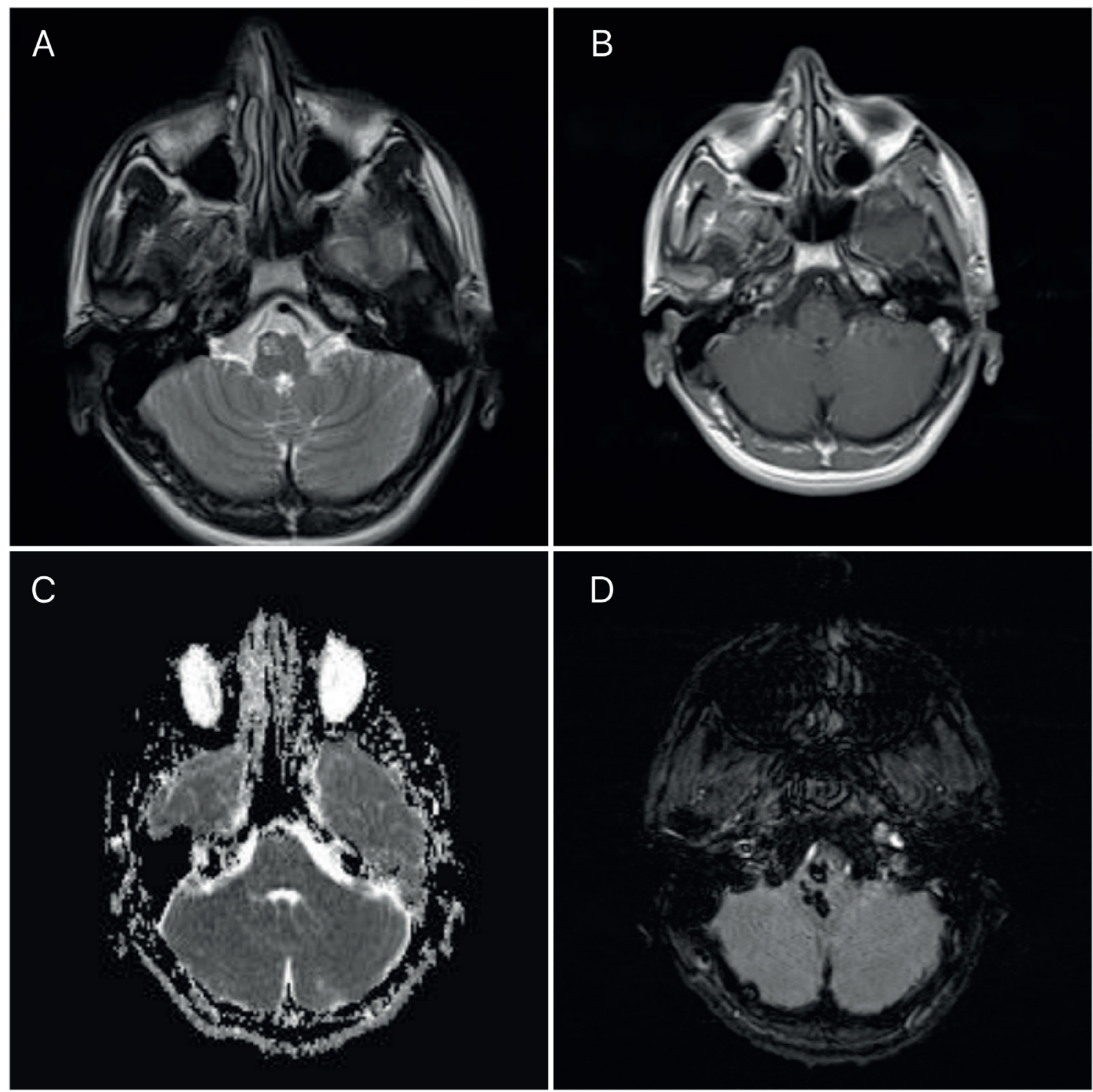

Fig. 1. a. Axial T2WI shows increased signal and enlargement of right inferior olivary nuclei. b. Axial T1WI shows hypointensity in right inferior olivary nuclei with no enhancement on contrast enhanced MRI. c. Axial apparent diffusion coefficient (ADC) image shows shows enlarged hyperintense inferior olivary nucleus with no diffusion restriction. d. SWI sequences shows a chronic hemorrhage involving the medulla oblongata and cerebellum.

chronic hemorrhage involving the medulla oblongata and cerebellum caused the ipsilateral HOD however the etiology of hemorrhage couldn't be found.

Persistent hiccups can indicate a number of different neurological diseases such as cerebral injury, encephalitis/meningitis, neuromyelitis optica, multiple sclerosis, or cerebrovascular diseases. Cranial MRI should be performed to determine the underlying neurological disease. ${ }^{13,14}$

In conclusion, HOD is a rare degenerative disorder that is thought to occur subsequent to a disruption of the dentate-rubro-olivary 
pathway. There is limited data in the literature about the clinical and radiological features of this disease in pediatric populations. We highlight a pediatric case of unilateral HOD, which presented with persistent hiccups. Hiccups was described in neuro-Behcet related to $\mathrm{HOD}^{15}$ however to our knowledge it has not been described in children with unilateral HOD in the literature. Awareness of clinical and radiological findings of HOD is important to avoid misinterpretation as a mass lesion, an ischemic event, or a demyelinating disease and provide adequate management.

\section{REFERENCES}

1. Gautier JG, Blackwood W. Enlargement of the inferior olivary nucleus in association with lesions of the central tegmental tract or dentate nucleus. Brain 1961; 84: 341-361.

2. Goyal M, Versnick E, Tuite P, et al. Hypertrophic olivary degeneration: metaanalysis of the temporal evolution of MR findings, AJNR Am J Neuroradiol 2000; 21: 1073-1077.

3. Ballestero MFM, Viana DC, Teixeira TL, Santos MV, de Oliveira RS. Hypertrophic olivary degeneration in children after posterior fossa surgery. An underdiagnosed condition. Childs Nerv Syst 2018; 34: 409-415.

4. Tartaglione T, Izzo G, Alexandre A, et al. MRI findings of olivary degeneration after surgery for posterior fossa tumours in children: incidence, time course and correlation with tumour grading. Radiol Med 2015; 120: 474-482.

5. Tilikete C, Desestret V. Hypertrophic olivary degeneration and palatal or oculopalatal tremor. Front Neurol 2017; 8: 302.
6. Walker G, Limaye K, Jankowitz BT, Jadhav AP Hypertrophic olivary degeneration after cerebellar hemorrhage. J Clin Neurosci 2017; 43: 162-164.

7. Nishie M, Yoshida Y, Hirata Y, Matsunaga M. Generation of symptomatic palatal tremor is not correlated with inferior olivary hypertrophy. Brain 2002; 125(Pt 6): 1348-1357.

8. Sanverdi SE, Oguz KK, Haliloglu G. Hypertrophic olivary degeneration in children: four new cases and a review of the literature with an emphasis on the MRI findings. Br J Radiol 2012; 85: 511-516.

9. Konno T, Broderick DF, Tacik P, Caviness JN, Wszolek ZK. Hypertrophic olivary degeneration: A clinico-radiologic study. Parkinsonism Relat Disord 2016; 28: 36-40.

10. Kinghorn KJ, Kaliakatsos M, Blakely EL, et al Hypertrophic olivary degeneration on magnetic resonance imaging in mitochondrial syndromes associated with POLG and SURF1 mutations. J Neurol 2013; 260: 3-9.

11. Oh SI. Hypertrophic olivary degeneration following listerial rhombencephalitis. Can J Neurol Sci 2018; 45: 593-595.

12. JI Seok, Yi H, Song YM, Lee WY. Metronidazoleinduced encephalopathy and inferior olivary hypertrophy: lesion analysis with diffusionweighted imaging and apparent diffusion coefficient maps. Arch Neurol 2003; 60: 1796-1800.

13. Eisenächer A, Spiske J. Persistent hiccups (singultus) as the presenting symptom of medullary cavernoma. Dtsch Arztebl Int 2011; 108: 822-826.

14. Sweeney J, Bodman A, Hall WA. Brain abscess of basal ganglia presenting with persistent hiccups. World Neurosurg 2018; 112: 182-185.

15. Franco-Macías E, Roldán-Lora F, Martínez-Agregado $\mathrm{P}$, Cerdá-Fuertes N, Moniche F. Neuro-Behçet: pons involvement with longitudinal extension to midbrain and hypertrophic olivary degeneration. Case Rep Neurol 2015; 7: 148-151. 